\title{
Positive solutions to fractional boundary value problems with nonlinear boundary conditions
}

Wenquan Feng, Shurong Sun ${ }^{*}$, Xinhui Li and Meirong Xu

\section{*Correspondence:} sshrong@163.com

School of Mathematical Sciences, University of Jinan, Jinan, Shandong 250022, P.R. China

\begin{abstract}
We consider the existence of at least one positive solution of the problem $-D_{0+}^{\alpha} u(t)=f(t, u(t)), 0<t<1$, under the circumstances that $u(0)=0$,

$u(1)=H_{1}(\varphi(u))+\int_{E} H_{2}(s, u(s)) d s$, where $1<\alpha<2, D_{0+}^{\alpha}$ is the Riemann-Liouville fractional derivative, and $u(1)=H_{1}(\varphi(u))+\int_{E} H_{2}(s, u(s)) d s$ represents a nonlinear nonlocal boundary condition. By imposing some relatively mild structural conditions on $f, H_{1}, H_{2}$, and $\varphi$, one positive solution to the problem is ensured. Our results generalize the existing results and an example is given as well.
\end{abstract}

MSC: $34 \mathrm{~A} 08 ; 34 \mathrm{~B} 18$

Keywords: fractional differential equation; nonlinear boundary condition; Krasnosel'skili's fixed point theorem; positive solution

\section{Introduction}

In this paper we consider the existence of at least one positive solution of the fractional differential equation

$$
-D_{0+}^{\alpha} u(t)=f(t, u(t)), \quad 0<t<1,
$$

subject to the boundary conditions

$$
u(0)=0, \quad u(1)=H_{1}(\varphi(u))+\int_{E} H_{2}(s, u(s)) d s,
$$

here $E \Subset(0,1)$ is some measurable set, $1<\alpha<2, D_{0+}^{\alpha}$ is the Riemann-Liouville fractional derivative and $\varphi$ is a linear functional having the form

$$
\varphi(u):=\int_{0}^{1} u(t) d \theta(t)
$$

where the integral appearing in (3) is taken in the Lebesgue-Stieltjes sense, $\theta$ is a function of bounded variation.

Let us review briefly some recent results on such problems in order to see our problem (1)-(2) in a more appropriate context. 
So far, in view of their various applications in science and engineering, such as fluid mechanics, control system, viscoelasticity, porous media, edge detection, optical systems, electromagnetism and so forth, see [1-15], fractional differential equations have attracted great attention of mathematicians.

There are a great number of works on the existence of solutions of various classes of ordinary differential equations and fractional differential equations; readers may refer to [16-32].

Some of them discussed two-point boundary value problems. For example, Bai and Lü [6] studied the following two-point boundary value problem of fractional differential equations:

$$
\begin{aligned}
& D_{0+}^{\alpha} u(t)+f(t, u(t))=0, \quad 0<t<1, \\
& u(0)=u(1)=0,
\end{aligned}
$$

where $1<\alpha \leq 2, D_{0+}^{\alpha}$ is the standard Riemann-Liouville fractional derivative. By means of Guo-Krasnosel'skii's fixed point theorem and the Leggett-Williams fixed point theorem they obtained the existence of positive solutions.

Some authors discussed multi-point boundary value problems, for instance, by using fixed point index theory, the Krein-Rutman theorem and some other methods, Jiang [7] studied the eigenvalue interval of the multi-point boundary value problem

$$
\begin{aligned}
& D^{\alpha} u(t)-M u(t)=\lambda f(t, u(t)), \quad t \in[0,1], \\
& u(0)=\sum_{i=1}^{n} \beta_{i} u\left(\xi_{i}\right),
\end{aligned}
$$

where $0<\alpha<1, D^{\alpha}$ is the Caputo derivative, $M \geq 0,0<\xi_{1}<\xi_{2}<\cdots<\xi_{n} \leq 1$.

There are also results on fractional boundary value problem with integral boundary conditions, let us refer to Vong [8]. He investigated positive solutions of the nonlocal boundary value problem for a class of singular fractional differential equations with an integral boundary condition,

$$
\begin{aligned}
& { }^{c} D_{0^{+}}^{\alpha} u(t)+f(t, u(t))=0, \\
& u^{\prime}(0)=\cdots=u^{(n-1)}(0)=0, \quad u(1)=\int_{0}^{1} u(s) d \mu(s),
\end{aligned}
$$

where $n \geq 2, \alpha \in(n-1, n)$ and $\mu$ is a function of bounded variation.

To proceed, Goodrich [9] considered the existence of at least one positive solution of the ordinary differential equation

$$
\begin{aligned}
& -y^{\prime \prime}(t)=f(t, y(t)), \quad 0<t<1, \\
& y(0)=H_{1}(\varphi(y))+\int_{E} H_{2}(s, y(s)) d s, \quad y(1)=0,
\end{aligned}
$$

in which the boundary condition is more general.

Motivated by the above works, we decided to consider the problem (1)-(2). As to the novel contributions of this work, we hold in the first place that the problem discussed in [9] is an ordinary differential equation, while we take a look into the frac- 
tional differential equation under the same boundary conditions. Secondly, the boundary conditions are more flexible and general than often. Let us take the condition $u(0)=$ $H_{1}(\varphi(u))+\int_{E} H_{2}(s, u(s)) d s$ into consideration, where $H_{1}, H_{2}, \varphi(u)$ are defined in the sequel. If $H_{1}(\varphi(u))+\int_{E} H_{2}(s, u(s)) d s=0$, the conditions are the standard Dirichlet boundary conditions. Readers might refer to Bai and Lü [6]. As far as we are concerned, $\varphi(u)$ varies among many sorts of functionals. If $H_{1}(\varphi(u))=\int_{F} u(t) d t$ (where $F \subset E \Subset(0,1)$ is defined in the sequel) or $H_{1}(\varphi(u))=\int_{[0,1]} u(t) d \theta(t)$, our conditions reduce to integral boundary conditions, while if $H_{1}(\varphi(u))=\sum_{i=1}^{n}\left|a_{i}\right| u\left(\xi_{i}\right)$, we have multi-point boundary conditions. Thirdly, compared to Goodrich [9], we make an adjustment to the Green function and define a function $r(\cdot)$ instead of a constant which affects the defined cone.

This paper is organized as follows. In Section 2, we review some preliminaries and lemmas. In Section 3, a theorem and five corollaries about the existence of at least one positive solution of problem (1)-(2) are obtained. Lastly, we give an example to illustrate the obtained theorem.

\section{Preliminaries and lemmas}

For the convenience of the readers, we give some background materials from fractional calculus theory to facilitate the analysis of the boundary value problem (1)-(2).

Definition 2.1 ([2]) The Riemann-Liouville fractional integral of order $\alpha>0$ of a function $y:(0,+\infty) \rightarrow \mathbb{R}$ is given by

$$
I_{0+}^{\alpha} y(t)=\frac{1}{\Gamma(\alpha)} \int_{0}^{t}(t-s)^{\alpha-1} y(s) d s
$$

provided the right side is pointwise defined on $(0,+\infty)$.

Definition 2.2 ([2]) The Riemann-Liouville typed fractional derivative of order $\alpha(\alpha>0)$ of a continuous function $f:(0,+\infty) \rightarrow \mathbb{R}$ is given by

$$
D_{0^{+}}^{\alpha} f(t)=\frac{1}{\Gamma(n-\alpha)}\left(\frac{d}{d t}\right)^{n} \int_{0}^{t}(t-s)^{n-\alpha+1} f(s) d s
$$

where $n=[\alpha]+1,[\alpha]$ denotes the integer part of number $\alpha$, provided that the right side is pointwise defined on $(0,+\infty)$.

Lemma 2.1 ([2]) Let $\alpha>0$. If we assume $u \in C(0,1) \cap L(0,1)$, then the fractional differential equation

$$
D_{0^{+}}^{\alpha} u(t)=0
$$

has $u(t)=c_{1} t^{\alpha-1}+c_{2} t^{\alpha-2}+\cdots+c_{n} t^{\alpha-n}, c_{i} \in \mathbb{R}, i=1,2, \ldots, n$, as a unique solution, where $n$ is the smallest integer greater than or equal to $\alpha$.

Lemma $2.2([2])$ Let $u \in C(0,1) \cap L(0,1)$ with a fractional derivative of order $\alpha(\alpha>0)$ that belongs to $C(0,1) \cap L(0,1)$. Then

$$
I_{0^{+}}^{\alpha} D_{0^{+}}^{\alpha} u(t)=u(t)+c_{1} t^{\alpha-1}+c_{2} t^{\alpha-2}+\cdots+c_{n} t^{\alpha-n}, \quad \text { for some } c_{i} \in \mathbb{R}, i=1,2, \ldots, n,
$$

where $n$ is the smallest integer greater than or equal to $\alpha$. 
Remark 2.1 ([2]) The Riemann-Liouville type fractional derivative and integral of order $\alpha(\alpha>0)$ have the following properties:

$$
D_{0^{+}}^{\alpha} I_{0^{+}}^{\alpha} u(t)=u(t), \quad I_{0^{+}}^{\alpha} I_{0^{+}}^{\beta} u(t)=I_{0^{+}}^{\alpha+\beta} u(t), \quad \alpha, \beta>0, u \in L(0,1) .
$$

Lemma 2.3 Let $u \in C[0,1]$ and $1<\alpha<2$. Then the fractional differential equation boundary value problem

$$
\begin{aligned}
& D_{0^{+}}^{\alpha} u(t)+y(t)=0, \quad 0<t<1, \\
& u(0)=0, \quad u(1)=H_{1}(\varphi(u))+\int_{E} H_{2}(s, u(s)) d s,
\end{aligned}
$$

has a unique solution,

$$
u(t)=t^{\alpha-1}\left[H_{1}(\varphi(u))+\int_{E} H_{2}(s, u(s)) d s\right]+\int_{0}^{1} G(t, s) y(s) d s,
$$

where

$$
G(t, s)=\frac{1}{\Gamma(\alpha)} \begin{cases}t^{\alpha-1}(1-s)^{\alpha-1}-(t-s)^{\alpha-1}, & 0 \leq s \leq t \leq 1 \\ t^{\alpha-1}(1-s)^{\alpha-1}, & 0 \leq t \leq s \leq 1\end{cases}
$$

Proof We may apply Lemma 2.2 to reduce (1) to an equivalent integral equation,

$$
u(t)=-I_{0_{+}}^{\alpha} y(s)+c_{1} t^{\alpha-1}+c_{2} t^{\alpha-2}, \quad c_{1}, c_{2} \in \mathbb{R} .
$$

Consequently, the general solution of (1) is

$$
u(t)=-\int_{0}^{t} \frac{(t-s)^{\alpha-1}}{\Gamma(\alpha)} y(s) d s+c_{1} t^{\alpha-1}+c_{2} t^{\alpha-2}, \quad c_{1}, c_{2} \in \mathbb{R} .
$$

By (2), we have

$$
\begin{aligned}
& c_{1}=H_{1}(\varphi(u))+\int_{E} H_{2}(s, u(s)) d s+\int_{0}^{1} \frac{(1-s)^{\alpha-1}}{\Gamma(\alpha)} y(s) d s, \\
& c_{2}=0 .
\end{aligned}
$$

Therefore, the unique solution of problem (1) and (2) is

$$
\begin{aligned}
u(t)= & t^{\alpha-1}\left[H_{1}(\varphi(u))+\int_{E} H_{2}(s, u(s)) d s\right] \\
& -\int_{0}^{t} \frac{(t-s)^{\alpha-1}}{\Gamma(\alpha)} y(s) d s+t^{\alpha-1} \int_{0}^{1} \frac{(1-s)^{\alpha-1}}{\Gamma(\alpha)} y(s) d s \\
= & t^{\alpha-1}\left[H_{1}(\varphi(u))+\int_{E} H_{2}(s, u(s)) d s\right]+\int_{0}^{1} G(t, s) y(s) d s .
\end{aligned}
$$

The proof is complete. 
Lemma 2.4 ([6]) Let $(a, b) \subset(0,1)$ be an arbitrary but fixed interval. Then the function $G(t, s)$ defined by (8) satisfies the following conditions:

(1) $G(t, s)>0$, for $t, s \in(0,1)$;

(2) there exists a positive function $\gamma(\cdot) \in C(0,1)$ such that

$$
\min _{a \leq t \leq b} G(t, s) \geq \gamma(s) \max _{0 \leq t \leq 1} G(t, s)=\gamma(s) G(s, s), \quad \text { for each } 0 \leq s \leq 1 .
$$

Lemma 2.5 ([4]) Let $B$ be a Banach space, and let $K \subset B$ be a cone. Assume $\Omega_{1}, \Omega_{2}$ are open and bounded subsets of $B$ with $0 \in \Omega_{1}, \bar{\Omega}_{1} \subset \Omega_{2}$, and let $T: K \cap\left(\bar{\Omega}_{2} \backslash \Omega_{1}\right) \rightarrow K$ be a completely continuous operator such that

(i) $\|T u\| \leq\|u\|, u \in K \cap \partial \Omega_{1}$, and $\|T u\| \geq\|u\|, u \in K \cap \partial \Omega_{2}$; or

(ii) $\|T u\| \geq\|u\|, u \in K \cap \partial \Omega_{1}$, and $\|T u\| \leq\|u\|, u \in K \cap \partial \Omega_{2}$.

Then $T$ has a fixed point in $K \cap\left(\bar{\Omega}_{2} \backslash \Omega_{1}\right)$.

In order to get the main results, we first need some structure on $H_{1}, H_{2}, \varphi$, and $f$ appearing in problem (1)-(2).

Let $B$ be the Banach space on $C([0,1])$ equipped with the usual supremum norm $\|\cdot\|$. Then define the cone $K \subseteq B$ by

$$
K=\left\{u \in B \mid u(t) \geq 0, \min _{a \leq t \leq b} u(t) \geq \gamma^{*}\|u\|, \varphi_{1}(u), \varphi_{2}(u) \geq 0\right\}
$$

where $\gamma^{*}=\min \left\{\min _{t \in[a, b]} t^{\alpha-1}, \min _{s \in[a, b]} \gamma(s)\right\}$.

Define the operator $T: C[0,1] \rightarrow C[0,1]$ by

$$
T u(t)=t^{\alpha-1}\left[H_{1}(\varphi(u))+\int_{E} H_{2}(s, u(s)) d s\right]+\int_{0}^{1} G(t, s) f(s, u(s)) d s .
$$

Here we come to the nine significant assumptions.

$\left(\mathrm{H}_{1}\right)$ Let $H_{1}:[0,+\infty) \rightarrow[0,+\infty)$ and $H_{2}:[0,1] \times[0,+\infty) \rightarrow[0,+\infty)$ be real-valued continuous functions.

$\left(\mathrm{H}_{2}\right)$ The functional $\varphi(u):=\int_{[0,1]} u(t) d \theta(t)$ can be written in the form

$$
\varphi(u)=\varphi_{1}(u)+\varphi_{2}(u):=\int_{[0,1]} u(t) d \theta_{1}(t)+\int_{[0,1]} u(t) d \theta_{2}(t)
$$

where $\theta, \theta_{1}, \theta_{2}:[0,1] \rightarrow \mathbb{R}$ satisfy $\theta, \theta_{1}, \theta_{2} \in B V([0,1])$, and $\varphi_{1}, \varphi_{2}$ are continuous linear functionals.

$\left(\mathrm{H}_{3}\right)$ There is a constant $C_{1} \in[0,1)$ such that the functional $\varphi$ in $(11)$ satisfies the inequality

$$
|\varphi(u)| \leq C_{1}\|u\|
$$

for all $u \in C([0,1])$. Furthermore, there is a constant $C_{2}>0$ such that the functional $\varphi_{2}$ in (11) satisfies $\left|\varphi_{2}(u)\right| \geq C_{2}\|u\|$ whenever $u \in K$.

$\left(\mathrm{H}_{4}\right)$ For each given $\varepsilon>0$, there are $C_{3}>0$ and $M_{\varepsilon}>0$ whenever $z \geq M_{\varepsilon}$ and we have

$$
\left|H_{1}(z)-C_{3} z\right|<\varepsilon C_{3} z
$$


$\left(\mathrm{H}_{5}\right)$ There exists a function $F:[0,+\infty) \rightarrow[0,+\infty)$ satisfying the growth condition

$$
F(u)<C_{4} u
$$

for some $C_{4} \geq 0$, having the property that for each given $\varepsilon>0$, there is $M_{\varepsilon}>0$ such that

$$
\left|H_{2}(x, u)-F(u)\right|<\varepsilon F(u)
$$

for all $x \in[0,1]$, whenever $u \geq M_{\varepsilon}$.

$\left(\mathrm{H}_{6}\right)$ Assume that the nonlinearity $f(t, u)$ splits in the sense that $f(t, u)=a(t) g(u)$, for continuous functions $a:[0,1] \rightarrow[0,+\infty)$ and $g: \mathbb{R} \rightarrow[0,+\infty)$ such that $a$ is not identically zero on any subinterval of $[0,1]$.

$\left(\mathrm{H}_{7}\right)$ Suppose $\lim _{u \rightarrow 0^{+}} \frac{g(u)}{u}=+\infty$.

$\left(\mathrm{H}_{8}\right)$ Suppose $\lim _{u \rightarrow+\infty} \frac{g(u)}{u}=0$.

$\left(\mathrm{H}_{9}\right)$ For each $i=1,2$ both

$$
\int_{[0,1]} t^{\alpha-1} d \theta_{i}(t) \geq 0
$$

and

$$
\int_{[0,1]} G(t, s) d \theta_{i}(t) \geq 0
$$

hold, where (17) holds for each $s \in[0,1]$.

\section{Main results}

In this section we state and prove the existence theorem of problem (1)-(2).

Lemma 3.1 Let $T$ be the operator defined in (10). Assume conditions $\left(\mathrm{H}_{1}\right)-\left(\mathrm{H}_{9}\right)$ hold. Then $T: K \rightarrow K$, and the operator $T$ is completely continuous.

Proof Now we divide the proof into two steps; in the first step we prove that $T: K \rightarrow K$, then in the next, the conclusion that the operator $T$ is completely continuous is treated.

Step 1 . Here we are going to show that $T: K \rightarrow K$. In fact, since $H_{1}, H_{2}, a$, and $g$ are all nonnegative, it is easy to find that whenever $u \in K$, it follows that $(T u)(t) \geq 0$, for each $t \in[0,1]$, where we use the fact $\varphi(u) \geq 0$, following $H_{1}(\varphi(u)) \geq 0$.

On the other hand, provided $u \in K$ we get

$$
\begin{aligned}
\min _{t \in[a, b]} T u(t) & \geq \min _{t \in[a, b]} t^{\alpha-1}\left[H_{1}(\varphi(u))+\int_{E} H_{2}(s, u(s)) d s\right]+\min _{t \in[a, b]} \int_{0}^{1} G(t, s) f(s, u(s)) d s \\
& \geq \gamma_{0}\left[H_{1}(\varphi(u))+\int_{E} H_{2}(s, u(s)) d s\right]+\max _{t \in[0,1]} \int_{0}^{1} \gamma(s) G(t, s) f(s, u(s)) d s \\
& \geq \gamma_{0}\left[H_{1}(\varphi(u))+\int_{E} H_{2}(s, u(s)) d s\right]+\max _{t \in[0,1]} \int_{a}^{b} \gamma(s) G(t, s) f(s, u(s)) d s \\
& \geq \gamma_{0}\left[H_{1}(\varphi(u))+\int_{E} H_{2}(s, u(s)) d s\right]
\end{aligned}
$$




$$
\begin{aligned}
& \quad+\min _{t \in[a, b]} \gamma(t) \max _{t \in[0,1]} \int_{a}^{b} G(t, s) f(s, u(s)) d s \\
& \geq \gamma^{*}\|T u\|,
\end{aligned}
$$

where $\gamma_{0}=\min _{t \in[a, b]} t^{\alpha-1}$.

Consequently, for each $i=1,2$ we deduce that

$$
\begin{aligned}
\varphi_{i}(T u)= & \int_{[0,1]} t^{\alpha-1}\left[H_{1}(\varphi(u))+\int_{E} H_{2}(s, u(s)) d s\right] d \theta_{i}(t) \\
& +\int_{[0,1]}\left(\int_{0}^{1} G(t, s) a(s) g(u(s)) d s\right) d \theta_{i}(t) \\
= & {\left[H_{1}(\varphi(u))+\int_{E} H_{2}(s, u(s)) d s\right] \int_{[0,1]} t^{\alpha-1} d \theta_{i}(t) } \\
& +\int_{0}^{1}\left[\int_{[0,1]} G(t, s) d \theta_{i}(t)\right] a(s) g(u(s)) d s \\
\geq & 0,
\end{aligned}
$$

where the inequality follows from assumption $\left(\mathrm{H}_{9}\right)$. Thus, $\varphi_{i}(\mathrm{Tu}) \geq 0$, therefore as desired we conclude $T(K) \subset K$.

Step 2. In this part we turn to the proof that $T(\Omega)$ in the sequel is bounded as well as equicontinuous with the help of the Arzelà-Ascoli theorem.

With the continuity of $H_{1}, H_{2}, a$, and $g$, it is easy to find $T$ is continuous.

Let $\Omega \subset K$ be bounded, namely, there exists a number $M>0$, such that for each $u \in \Omega$ we have $\|u\| \leq M$. By the continuity of $H_{1}, H_{2}$, and $\varphi$, we find $H_{1}, H_{2}$ are bounded, so there exist constants $P>0$ and $Q>0$ such that $\left|H_{1}(\varphi(\Omega))\right| \leq P$ and $\left|H_{2}(t, u(t))\right| \leq Q . E \Subset(0,1)$ is a measurable set, take $L=\max _{t \in[0,1], u \in[0, M]}|f(t, u)|+1$, then

$$
\begin{aligned}
|(T u)(t)| & =\left|t^{\alpha-1}\left[H_{1}(\varphi(u))+\int_{E} H_{2}(s, u(s)) d s\right]+\int_{0}^{1} G(t, s) f(s, u(s)) d s\right| \\
& \leq\left|H_{1}(\varphi(u))\right|+\int_{E}\left|H_{2}(s, u(s))\right| d s+\int_{0}^{1} G(t, s)|f(s, u(s))| d s \\
& \leq P+Q m(E)+L \int_{0}^{1} G(t, s) d s \\
& \leq P+Q m(E)+L \int_{0}^{1} G(s, s) d s \\
& =P+Q m(E)+\frac{L}{\Gamma(\alpha+2)} .
\end{aligned}
$$

Hence $T(\Omega)$ is bounded.

For each $u \in \Omega, t_{1}, t_{2} \in[0,1], t_{1}<t_{2}$, and assuming there is a $\delta>0$, such that $t_{2}-t_{1}<\delta$, for each $\varepsilon>0$ put

$$
\delta=\min \left\{\varepsilon, \frac{1}{2}\left(\frac{\varepsilon}{2 N}\right)^{\frac{1}{\alpha-1}},\left[\frac{\varepsilon}{2(\alpha-1) N}\right]^{\frac{1}{\alpha-1}},\left(\frac{\varepsilon}{2 N \alpha}\right)^{\frac{1}{\alpha}}\right\},
$$


where $N=P+Q m(E)+\frac{L}{\Gamma(\alpha+1)}$, we find $\left|(T u)\left(t_{2}\right)-(T u)\left(t_{1}\right)\right|<\varepsilon$, so that $T(\Omega)$ is equicontinuous.

Indeed,

$$
\begin{aligned}
\left|(T u)\left(t_{2}\right)-(T u)\left(t_{1}\right)\right| \\
=\mid t_{2}^{\alpha-1}\left[H_{1}(\varphi(u))+\int_{E} H_{2}(s, u(s)) d s\right]+\int_{0}^{1} G\left(t_{2}, s\right) f(s, u(s)) d s \\
\quad-t_{1}^{\alpha-1}\left[H_{1}(\varphi(u))+\int_{E} H_{2}(s, u(s)) d s\right]-\int_{0}^{1} G\left(t_{1}, s\right) f(s, u(s)) d s \mid \\
=\left|\left(t_{2}^{\alpha-1}-t_{1}^{\alpha-1}\right)\left[H_{1}(\varphi(u))+\int_{E} H_{2}(s, u(s)) d s\right]+\int_{0}^{1}\left(G\left(t_{2}, s\right)-G\left(t_{1}, s\right)\right) f(s, u(s)) d s\right| \\
\leq\left(t_{2}^{\alpha-1}-t_{1}^{\alpha-1}\right)\left[\left|H_{1}(\varphi(u))\right|+\int_{E}\left|H_{2}(s, u(s))\right| d s\right] \\
\quad+\left|\int_{0}^{1}\left(G\left(t_{2}, s\right)-G\left(t_{1}, s\right)\right) f(s, u(s)) d s\right| .
\end{aligned}
$$

By the continuity of $H_{1}, H_{2}$, and $\varphi$, we find $H_{1}, H_{2}$ are bounded, so there exist constants $P>0$ and $Q>0$ such that $\left|H_{1}(\varphi(\Omega))\right| \leq P$ and $\left|H_{2}(t, u(t))\right| \leq Q . E \Subset(0,1)$ is a measurable set, take $L=\max _{t \in[0,1], u \in[0, M]}|f(t, u)|+1$, then

$$
\begin{aligned}
\left|(T u)\left(t_{2}\right)-(T u)\left(t_{1}\right)\right| & \\
\leq & \left(t_{2}^{\alpha-1}-t_{1}^{\alpha-1}\right)[P+Q m(E)]+\int_{0}^{1}\left|\left(G\left(t_{2}, s\right)-G\left(t_{1}, s\right)\right) f(s, u(s))\right| d s \\
\leq & \left(t_{2}^{\alpha-1}-t_{1}^{\alpha-1}\right)[P+Q m(E)] \\
& +\frac{L}{\Gamma(\alpha)}\left[\int_{0}^{t_{1}}\left|t_{2}^{\alpha-1}(1-s)^{\alpha-1}-\left(t_{2}-s\right)^{\alpha-1}-t_{1}^{\alpha-1}(1-s)^{\alpha-1}+\left(t_{1}-s\right)^{\alpha-1}\right| d s\right. \\
& +\int_{t_{1}}^{t_{2}}\left|t_{2}^{\alpha-1}(1-s)^{\alpha-1}-\left(t_{2}-s\right)^{\alpha-1}-t_{1}^{\alpha-1}(1-s)^{\alpha-1}\right| d s \\
& \left.+\int_{t_{2}}^{1}\left|t_{2}^{\alpha-1}(1-s)^{\alpha-1}-t_{1}^{\alpha-1}(1-s)^{\alpha-1}\right| d s\right] \\
\leq & \left(t_{2}^{\alpha-1}-t_{1}^{\alpha-1}\right)[P+Q m(E)]+\frac{L}{\Gamma(\alpha)}\left\{\left|t_{2}^{\alpha-1}-t_{1}^{\alpha-1}\right| \int_{0}^{t_{1}}(1-s)^{\alpha-1} d s\right. \\
& +\int_{0}^{t_{1}}\left[\left(t_{2}-s\right)^{\alpha-1}-\left(t_{1}-s\right)^{\alpha-1}\right] d s+\left|t_{2}^{\alpha-1}-t_{1}^{\alpha-1}\right| \int_{t_{1}}^{t_{2}}(1-s)^{\alpha-1} d s \\
& \left.+\int_{t_{1}}^{t_{2}}\left(t_{2}-s\right)^{\alpha-1} d s+\left|t_{2}^{\alpha-1}-t_{1}^{\alpha-1}\right| \int_{t_{2}}^{1}(1-s)^{\alpha-1} d s\right\} \\
= & \left(t_{2}^{\alpha-1}-t_{1}^{\alpha-1}\right)\left[P+Q m(E)+\frac{L}{\Gamma(\alpha+1)}\right]+\frac{L}{\Gamma(\alpha+1)}\left(t_{2}^{\alpha}-t_{1}^{\alpha}\right) .
\end{aligned}
$$

Next we discuss the following three cases.

Case $1 . \delta \leq t_{1}<t_{2}<1$.

$$
\begin{aligned}
& t_{2}^{\alpha-1}-t_{1}^{\alpha-1} \leq \frac{\alpha-1}{\delta^{2-\alpha}}\left(t_{2}-t_{1}\right) \leq(\alpha-1) \delta^{\alpha-1} \leq \frac{\varepsilon}{2 N}, \\
& t_{2}^{\alpha}-t_{1}^{\alpha} \leq \frac{\alpha}{\delta^{1-\alpha}}\left(t_{2}-t_{1}\right) \leq \alpha \delta^{\alpha} \leq \frac{\varepsilon}{2 N}
\end{aligned}
$$


Case $2.0 \leq t_{1}<\delta<t_{2}<2 \delta$.

$$
\begin{aligned}
& t_{2}^{\alpha-1}-t_{1}^{\alpha-1} \leq t_{2}^{\alpha-1} \leq(2 \delta)^{\alpha-1} \leq \frac{\varepsilon}{2 N} \\
& t_{2}^{\alpha}-t_{1}^{\alpha} \leq t_{2}^{\alpha} \leq(2 \delta)^{\alpha} \leq \frac{\varepsilon}{2 N}
\end{aligned}
$$

Case 3. $0 \leq t_{1}<t_{2}<\delta$.

$$
\begin{aligned}
& t_{2}^{\alpha-1}-t_{1}^{\alpha-1} \leq t_{2}^{\alpha-1} \leq \delta^{\alpha-1} \leq \frac{\varepsilon}{2 N} \\
& t_{2}^{\alpha}-t_{1}^{\alpha} \leq t_{2}^{\alpha} \leq \delta^{\alpha} \leq \frac{\varepsilon}{2 N}
\end{aligned}
$$

Hence $\left|(T u)\left(t_{2}\right)-(T u)\left(t_{1}\right)\right| \leq \varepsilon$.

From the Arzelà-Ascoli theorem, $T$ is completely continuous. The proof is complete.

With Lemma 3.1 in hand, we are now ready to present the first existence theorem for problem (1) and (2).

Theorem 3.1 Assume that conditions $\left(\mathrm{H}_{1}\right)-\left(\mathrm{H}_{9}\right)$ hold. Suppose that

$$
C_{1} C_{3}+C_{4} m(E)<1
$$

and that $E \Subset(0,1)$. Then problem (1)-(2) has at least one positive solution.

Proof Begin by selecting a number $\eta_{1}$ such that

$$
\eta_{1} \int_{a}^{b} \gamma^{*} G\left(\frac{1}{2}, s\right) a(s) d s \geq 1
$$

From $\left(\mathrm{H}_{7}\right)$, there exists a number $r_{1}>0$ such that $g(u) \geq \eta_{1} u$ whenever $0<u \leq r_{1}$. Then set

$$
\Omega_{r_{1}}:=\left\{u \in B:\|u\|<r_{1}\right\}
$$

for $u \in K \cap \partial \Omega_{r_{1}}$ we find

$$
\begin{aligned}
(T u)\left(\frac{1}{2}\right) & \geq \int_{0}^{1} G\left(\frac{1}{2}, s\right) a(s) g(u(s)) d s \geq\|u\| \eta_{1} \int_{a}^{b} \gamma^{*} G\left(\frac{1}{2}, s\right) a(s) d s \\
& \geq\|u\|,
\end{aligned}
$$

from the definition of norm $\|\cdot\|$, we get $\|T u\| \geq\|u\|$, and so, $T$ is a cone expansion on $K \cap \partial \Omega_{r_{1}}$. 
From condition (27), we choose a positive number $\varepsilon$ small enough, so that we may assume

$$
C_{1} C_{3} \varepsilon+C_{1} C_{3}+\varepsilon+\left(C_{4}+C_{4} \varepsilon\right) m(E) \leq 1 .
$$

Since $\varphi_{1}(u) \geq 0$, it follows that $\varphi(u) \geq \varphi_{2}(u) \geq C_{2}\|u\|$, if $\|u\| \geq \frac{M_{\varepsilon}}{C_{2}}$, then $\varphi(u)>M_{\varepsilon}$, and then by condition $\left(\mathrm{H}_{4}\right)$, we have

$$
\left|H_{1}(\varphi(u))-C_{3} \varphi(u)\right|<\varepsilon C_{3} \varphi(u) .
$$

For $E \Subset(0,1)$, we may select $0<a<b<1$ such that $E \subseteq(a, b)$. By the definition of the cone, we have

$$
\min _{t \in E} u(t) \geq \min _{t \in[a, b]} u(t) \geq r^{*}\|u\|
$$

If $\|u\| \geq \frac{M_{\varepsilon}}{r^{*}}$, then

$$
\min _{t \in E} u(t) \geq \min _{t \in[a, b]} u(t) \geq r^{*}\|u\| \geq r^{*} \frac{M_{\varepsilon}}{r^{*}}=M_{\varepsilon} .
$$

Hence, by condition $\left(\mathrm{H}_{5}\right)$ we get

$$
\left|H_{2}(x, u(s))-F(u(s))\right|<\varepsilon F(u(s)) .
$$

Provided that

$$
\|u\| \geq \max \left\{\frac{M_{\varepsilon}}{C_{2}}, \frac{M_{\varepsilon}}{r^{*}}\right\},
$$

both (32) and (35) hold.

Next we are going to discuss these two cases: $g$ is bounded and unbounded on $[0,+\infty)$, respectively.

Now if $g$ is bounded on $[0,+\infty)$, then there exists $r_{2}>0$ sufficiently large such that

$$
g(u) \leq r_{2}, \quad \text { for any } u \geq 0 .
$$

Indeed, we might assume without loss of generality that

$$
g(u) \leq \frac{r_{2} \varepsilon}{\int_{0}^{1} G(s, s) a(s) d s},
$$

where $\varepsilon$ is selected sufficiently small such that both (32) and (35) hold. We define a number

$$
r_{2}^{*}:=\max \left\{\frac{2 r_{1}}{r^{*}}, r_{2}, \frac{M_{\varepsilon}}{C_{2}}, \frac{M_{\varepsilon}}{r^{*}}\right\} .
$$

Set

$$
\Omega_{r_{2}^{*}}:=\left\{u \in B:\|u\|<r_{2}^{*}\right\} .
$$


Then for each $u \in K \cap \partial \Omega_{r_{2}^{*}}$ we find that

$$
\begin{aligned}
\|T u\| \leq & H_{1}(\varphi(u))+\int_{E} H_{2}(s, u(s)) d s+\int_{0}^{1} G(s, s) a(s) g(u(s)) d s \\
\leq & \left|H_{1}(\varphi(u))-C_{3} \varphi(u)\right|+C_{3}|\varphi(u)|+\int_{E}\left|H_{2}(s, u(s))-F(u(s))\right| d s \\
& +\int_{E}|F(u(s))| d s+\int_{0}^{1} r_{2} \varepsilon d s \\
\leq & \varepsilon C_{1} C_{3}\|u\|+C_{1} C_{3}\|u\|+m(E) C_{4}(1+\varepsilon)\|u\|+\varepsilon\|u\| \\
= & {\left[C_{1} C_{3} \varepsilon+C_{1} C_{3}+m(E) C_{4}(1+\varepsilon)+\varepsilon\right]\|u\| } \\
\leq & \|u\|
\end{aligned}
$$

whence $T$ is a cone compression on $K \cap \partial \Omega_{r_{2}^{*}}$.

On the other hand, assume $g$ is unbounded on $[0,+\infty)$. From condition $\left(\mathrm{H}_{8}\right)$ there is a number $r_{3}>0$ such that $g(u) \leq \eta_{2} u$ whenever $u>r_{3}$, where $\eta_{2}$ is picked with

$$
\eta_{2} \int_{0}^{1} G(s, s) a(s) d s \leq \varepsilon .
$$

Since $g$ is unbounded on $[0,+\infty)$, we can find a number $r_{3}^{*}$ satisfying

$$
r_{3}^{*}>\max \left\{\frac{2 r_{1}}{r^{*}}, r_{3}, \frac{M_{\varepsilon}}{C_{2}}, \frac{M_{\varepsilon}}{r^{*}}\right\},
$$

such that $g(u) \leq g\left(r_{3}^{*}\right)$ for any $u \in\left[0, r_{3}^{*}\right]$.

Take

$$
\Omega_{r_{3}^{*}}:=\left\{u \in B:\|u\|<r_{3}^{*}\right\}
$$

Then for each $u \in K \cap \partial \Omega_{r_{3}^{*}}$ we find that

$$
\begin{aligned}
\|T u\| \leq & H_{1}(\varphi(u))+\int_{E} H_{2}(s, u(s)) d s+\int_{0}^{1} G(s, s) a(s) g(u(s)) d s \\
\leq & \left|H_{1}(\varphi(u))-C_{3} \varphi(u)\right|+C_{3}|\varphi(u)|+\int_{E}\left|H_{2}(s, u(s))-F(u(s))\right| d s \\
& +\int_{E}|F(u(s))| d s+\int_{0}^{1} G(s, s) a(s) g\left(r_{3}^{*}\right) d s \\
\leq & C_{3} \varepsilon \varphi(u)+C_{1} C_{3}\|u\|+\int_{E}(1+\varepsilon) F(u(s)) d s+\int_{0}^{1} G(s, s) a(s) g\left(r_{3}^{*}\right) d s \\
\leq & \varepsilon C_{1} C_{3}\|u\|+C_{1} C_{3}\|u\|+m(E) C_{4}(1+\varepsilon)\|u\|+\varepsilon\|u\| \\
= & {\left[C_{1} C_{3} \varepsilon+C_{1} C_{3}+m(E) C_{4}(1+\varepsilon)+\varepsilon\right]\|u\| } \\
\leq & \|u\|,
\end{aligned}
$$

whence $T$ is a cone compression on $K \cap \partial \Omega_{r_{3}^{*}}$. 
Therefore, in either case, define $r_{4}^{*}=\max \left\{r_{2}^{*}, r_{3}^{*}\right\}$, we find $\|T u\| \leq\|u\|$ whenever $u \in K \cap$ $\partial \Omega_{r_{4}^{*}}$. From Lemma 2.5, we claim that problem (1)-(2) has at least one positive solution; the proof is complete.

Next we are going to give some corollaries since $\varphi(u)$ admits a wide variety of functionals. First we assume $H_{1}(\varphi(u))=0$, then, respectively, that $H_{1}(\varphi(u))=\int_{F} u(t) d t, H_{1}(\varphi(u))=$ $\sum_{i=1}^{n}\left|a_{i}\right| u\left(\xi_{i}\right)$ and $H_{1}(\varphi(u))=\int_{[0,1]} u(t) d \theta(t)$, where $F \subset E \Subset(0,1)$ is not Lebesgue null. In addition, we know $u(0)=0$ is also well defined and if $u(0)=0$ the problem as well as the boundary conditions are similar to [6].

Corollary 3.1 Assume that conditions $\left(\mathrm{H}_{1}\right),\left(\mathrm{H}_{6}\right)-\left(\mathrm{H}_{9}\right)$ hold, then the problem with Dirichlet conditions

$$
\begin{aligned}
& -D_{0+}^{\alpha} u(t)=f(t, u(t)), \quad 0<t<1, \\
& u(0)=0, \quad u(1)=0,
\end{aligned}
$$

has at least one positive solution.

Corollary 3.2 Assume that conditions $\left(\mathrm{H}_{1}\right)-\left(\mathrm{H}_{9}\right)$ hold. Suppose, in addition,

$$
C_{1} C_{3}+C_{4} m(E)<1
$$

and $F \subset E \Subset(0,1)$ is not Lebesgue null, and $m(F) \leq C_{1}$, then the problem with integral conditions

$$
\begin{aligned}
& -D_{0+}^{\alpha} u(t)=f(t, u(t)), \quad 0<t<1, \\
& u(1)=\int_{F} u(t) d t+\int_{E} H_{2}(s, u(s)) d s, \quad u(0)=0,
\end{aligned}
$$

has at least one positive solution.

Corollary 3.3 Assume that conditions $\left(\mathrm{H}_{1}\right)-\left(\mathrm{H}_{9}\right)$ hold. Suppose, in addition,

$$
C_{1} C_{3}+C_{4} m(E)<1
$$

and $E \Subset(0,1)$ is not Lebesgue null, and $\sum_{i=1}^{n}\left|a_{i}\right| \leq C_{1}$, then the problem with multi-point conditions

$$
\begin{aligned}
& -D_{0+}^{\alpha} u(t)=f(t, u(t)), \quad 0<t<1, \\
& u(1)=\sum_{i=1}^{n}\left|a_{i}\right| u\left(\xi_{i}\right)+\int_{E} H_{2}(s, u(s)) d s, \quad u(0)=0,
\end{aligned}
$$

has at least one positive solution.

Corollary 3.4 Assume that conditions $\left(\mathrm{H}_{1}\right)-\left(\mathrm{H}_{9}\right)$ hold. Suppose, in addition,

$$
C_{1} C_{3}+C_{4} m(E)<1
$$


and $E \Subset(0,1)$ is not Lebesgue null, and the total variation of $\theta$ over $[0,1]$ satisfies $V_{[0,1]}(\theta) \leq$ $C_{1}$, then the problem with the Lebesgue-Stieltjes integral conditions

$$
\begin{aligned}
& -D_{0+}^{\alpha} u(t)=f(t, u(t)), \quad 0<t<1, \\
& u(1)=\int_{[0,1]} u(t) d \theta(t)+\int_{E} H_{2}(s, u(s)) d s, \quad u(0)=0,
\end{aligned}
$$

has at least one positive solution.

Specially, take $\theta(t)=t^{2}-t$, for any $x, y \in[0,1]$ we have

$$
|\theta(x)-\theta(y)|=|(x-y)(x+y-1)| \leq|x-y|
$$

then

$$
V_{[0,1]}(\theta)=\sum_{i=1}^{n}\left|\theta\left(x_{i}\right)-\theta\left(x_{i-1}\right)\right| \leq \sum_{i=1}^{n}\left|x_{i}-x_{i-1}\right|=\sum_{i=1}^{n}\left(x_{i}-x_{i-1}\right)=1,
$$

thus we have the following corollary.

Corollary 3.5 Assume that conditions $\left(\mathrm{H}_{1}\right)-\left(\mathrm{H}_{4}\right)$ and $\left(\mathrm{H}_{6}\right)-\left(\mathrm{H}_{9}\right)$ hold. Suppose, in addition,

$$
C_{1} C_{3}<1,
$$

then the problem

$$
\begin{aligned}
& -D_{0+}^{\alpha} u(t)=f(t, u(t)), \quad 0<t<1, \\
& u(1)=\int_{[0,1]} u(t) d\left(t^{2}-t\right), \quad u(0)=0,
\end{aligned}
$$

has at least one positive solution.

\section{Example}

In this part we give an example of Theorem 3.1.

Define $\varphi(u)$ in the first place by

$$
\varphi(u)=\frac{1}{5} u\left(\frac{3}{4}\right)-\frac{1}{4} u\left(\frac{2}{5}\right)+\int_{\left[\frac{3}{10}, \frac{2}{5}\right]} u(t) d t
$$

where

$$
\varphi_{1}(u)=\frac{1}{5} u\left(\frac{3}{4}\right)-\frac{1}{4} u\left(\frac{2}{5}\right), \quad \varphi_{2}(u)=\int_{\left[\frac{3}{10}, \frac{2}{5}\right]} u(t) d t
$$

Then define $H_{1}, H_{2}$ by

$$
H_{1}(z)=\ln (z+1)+z
$$


and

$$
H_{2}(x, u)=3 u+2 x^{2}+e^{x} \sqrt{u} .
$$

It is clear that

$$
\lim _{z \rightarrow \infty}|(\ln (z+1)+z)-z|=0
$$

and moreover

$$
\lim _{u \rightarrow \infty} \frac{\left|\left(3 u+2 x^{2}+e^{x} \sqrt{u}\right)-3 u\right|}{3 u}=0,
$$

so conditions $\left(\mathrm{H}_{4}\right)$ and $\left(\mathrm{H}_{5}\right)$ hold, and we see $C_{3}=1, C_{4}=3$, and $F(u)=3 u$.

Now we consider the boundary value problem

$$
\begin{aligned}
& -D_{0+}^{\alpha} u(t)=f(t, u(t)), \quad 0<t<1, \\
& u(1)=\ln (\varphi(u)+1)+\varphi(u)+\int_{\frac{7}{20}}^{\frac{2}{5}}\left[3 u(s)+2 s^{2}+e^{s} \sqrt{u(s)}\right] d s, \quad u(0)=0,
\end{aligned}
$$

where $f(t, u(t))$ is a given function with conditions $\left(\mathrm{H}_{7}\right)$ and $\left(\mathrm{H}_{8}\right)$ satisfied. Here $E=\left[\frac{7}{20}, \frac{2}{5}\right]$ is chosen such that $m(E)=\frac{1}{20}$ and $E \subset(0,1)$.

What is more, for each $u \in K$

$$
|\varphi(u)| \leq \frac{1}{5}\|u\|+\frac{1}{4}\|u\|+\left(\frac{2}{5}-\frac{3}{10}\right)\|u\|=\frac{11}{20}\|u\|
$$

and

$$
\left|\varphi_{2}(u)\right| \geq \frac{1}{10} r^{*}\|u\|
$$

Then we find that $C_{1}=\frac{11}{20} \in[0,1]$ and $C_{2}=\frac{1}{10} r^{*}>0$, so condition $\left(\mathrm{H}_{3}\right)$ is met as well. Finally, after straightforward numerical calculations, condition $\left(\mathrm{H}_{9}\right)$ can also be achieved, since

$$
C_{1} C_{3}+C_{4} m(E)=\frac{11}{20} \cdot 1+3 \cdot \frac{1}{20}<1 .
$$

As a consequence, each of conditions $\left(\mathrm{H}_{1}\right)-\left(\mathrm{H}_{9}\right)$ is satisfied. From Theorem 3.1, problem (55) has at least one positive solution.

Competing interests

The authors declare that they have no competing interests.

Authors' contributions

All authors contributed equally to the writing of this paper. All authors read and approved the final manuscript.

\section{Acknowledgements}

The authors sincerely thank the reviewers for their valuable suggestions and useful comments, which have led to the present improved version of the original manuscript. This research is supported by the Natural Science Foundation of China (61374074), Natural Science Outstanding Youth Foundation of Shandong Province (JQ201119) and supported by Shandong Provincial Natural Science Foundation (ZR2012AM009, ZR2013AL003), also supported by Graduate Innovation Foundation of University of Jinan (YCX13013). 


\section{References}

1. Oldham, KB, Spanier, J: The Fractional Calculus. Academic Press, New York (1974)

2. Kilbas, AA, Srivastava, HH, Trujillo, JJ: Theory and Applications of Fractional Differential Equations. Elsevier, Amsterdam (2006)

3. Hardy, GH, Littlewood, JE, Pólya, G: Inequalities. Reprint of the 1952 Edition. Cambridge University Press, Cambridge (1988)

4. Guo, D, Lakshmikantham, V: Nonlinear Problems in Abstract Cones. Academic Press, Orlando (1988)

5. Podlubny, I: Fractional Differential Equations. Academic Press, New York (1999)

6. Bai, Z, Lü, H: Positive solutions for boundary value problem of nonlinear fractional differential equation. J. Math. Anal. Appl. 311, 495-505 (2005)

7. Jiang, W: Eigenvalue interval for multi-point boundary value problems of fractional differential equations. Appl. Math. Comput. 219, 4570-4575 (2013)

8. Vong, S: Positive solutions of singular fractional differential equations with integral boundary conditions. Math. Comput. Model. 57, 1053-1059 (2013)

9. Goodrich, CS: On a nonlocal BVP with nonlinear boundary conditions. Results Math. 63, 1351-1364 (2013)

10. Anguraj, A, Karthikeyan, P, Rivero, M, Trujillo, JJ: On new existence results for fractional integro-differential equations with impulsive and integral conditions. Comput. Math. Appl. 66, 2587-2594 (2014)

11. Cabada, A, Wang, G: Positive solutions of nonlinear fractional differential equations with integral boundary value conditions. J. Math. Anal. Appl. 389, 403-411 (2012)

12. Chalishajar, DN, Karthikeyan, K: Existence and uniqueness results for boundary value problems of higher order fractional integro-differential equations involving Gronwall's inequality in Banach spaces. Acta Math. Sci. 33, 758-772 (2013)

13. Zhang, L, Ahmad, B, Wang, G, Agarwal, R: Nonlinear fractional integro-differential equations on unbounded domains in a Banach space. J. Comput. Appl. Math. 249, 51-56 (2013)

14. Zhang, S: Positive solutions for boundary-value problems of nonlinear fractional differential equations. Electron. J. Differ. Equ. 2006, 36 (2006)

15. Sun, $\mathrm{S}, \mathrm{Li}, \mathrm{Q}, \mathrm{Li}, \mathrm{Y}$ : Existence and uniqueness of solutions for a coupled system of multi-term nonlinear fractional differential equations. Comput. Math. Appl. 64, 3310-3320 (2012)

16. Liang, S, Zhang, J: Positive solutions for boundary value problems of nonlinear fractional differential equation. Nonlinear Anal. 71, 5545-5550 (2009)

17. Li, N, Wang, C: New existence results of positive solution for a class of nonlinear fractional differential equations. Acta Math. Sci. 33(3), 847-854 (2013)

18. Shi, A, Zhang, S: Upper and lower solutions method and a fractional differential equation boundary value problem. Electron. J. Qual. Theory Differ. Equ. 2009, 30 (2009)

19. Su, X: Boundary value problem for a coupled system of nonlinear fractional differential equations. Appl. Math. Lett. 22, 64-69 (2009)

20. Benchohra, M, Hamani, S, Ntouyas, SK: Boundary value problems for differential equations with fractional order and nonlocal conditions. Nonlinear Anal. 71, 2391-2396 (2009)

21. Feng, W, Sun, S, Han, Z, Zhao, Y: Existence of solutions for a singular system of nonlinear fractional differential equations. Comput. Math. Appl. 62, 1370-1378 (2011)

22. Sun, S, Zhao, Y, Han, Z, Xu, M: Uniqueness of positive solutions for boundary value problems of singular fractional differential equations. Inverse Probl. Sci. Eng. 20, 299-309 (2012)

23. Stanĕk, S: The existence of positive solutions of singular fractional boundary value problems. Comput. Math. Appl. 62, 1379-1388 (2011)

24. Bai, C, Fang, J: The existence of a positive solution for a singular coupled system of nonlinear fractional differential equations. Appl. Math. Comput. 150, 611-621 (2004)

25. Zhang, Q, Jiang, D: Upper and lower solutions method and a second order three-point singular boundary value problem. Comput. Math. Appl. 56, 1059-1070 (2008)

26. Jiang, D: Upper and lower solutions method and a superlinear singular boundary value problem. Comput. Math. Appl. 44, 323-337 (2002)

27. Zhao, Y, Sun, S, Han, Z, Li, Q: The existence of multiple positive solutions for boundary value problems of nonlinear fractional differential equations. Commun. Nonlinear Sci. Numer. Simul. 16, 2086-2097 (2011)

28. Zhao, Y, Sun, S, Han, Z, Li, Q: Theory of fractional hybrid differential equations. Comput. Math. Appl. 62, 1312-1324 (2011)

29. Sun, S, Zhao, Y, Han, Z, Li, Y: The existence of solutions for boundary value problem of fractional hybrid differential equations. Commun. Nonlinear Sci. Numer. Simul. 17, 4961-4967 (2012)

30. Ding, $X$, Feng, $Y, B$, R: Existence nonexistence and multiplicity of positive solutions for nonlinear fractional differentia equations. J. Appl. Math. Comput. 40, 371-381 (2012)

31. Yan, R, Sun, S, Sun, Y, Han, Z: Boundary value problems for fractional differential equations with nonlocal boundary conditions. Adv. Differ. Equ. 2013, 176 (2013)

32. Li, X, Han, Z, Sun, S: Existence of positive solutions of nonlinear fractional q-difference equation with parameter. Adv. Differ. Equ. 2013, 260 (2013)

doi:10.1186/s13661-014-0225-0

Cite this article as: Feng et al.: Positive solutions to fractional boundary value problems with nonlinear boundary

conditions. Boundary Value Problems 2014 2014:225. 